The University of San Francisco

USF Scholarship: a digital repository@ Gleeson Library |

Geschke Center

Mathematics

College of Arts and Sciences

2006

\title{
Spectral Analysis of the Supreme Court
}

B Lawson

M Orrison

David Uminsky

University of San Francisco, duminsky@usfca.edu

Follow this and additional works at: http://repository.usfca.edu/math

Part of the Mathematics Commons

\section{Recommended Citation}

Lawson, B., Orrison, M., \& Uminsky, D. (2006). Spectral analysis of the supreme court. Mathematics Magazine, 79(5), $340-346$. http://dx.doi.org/10.2307/27642969

This Article is brought to you for free and open access by the College of Arts and Sciences at USF Scholarship: a digital repository @ Gleeson Library | Geschke Center. It has been accepted for inclusion in Mathematics by an authorized administrator of USF Scholarship: a digital repository @ Gleeson Library $\mid$ Geschke Center. For more information, please contact repository@usfca.edu. 
This article will appear in Mathematics Magazine.

\title{
Spectral Analysis of the Supreme Court
}

\author{
Brian L. Lawson \\ Department of Political Science \\ University of Cincinnati \\ Cincinnati, OH 45221 \\ brian. lawson@uc.edu \\ Michael E. Orrison \\ Harvey Mudd College \\ Claremont, CA 91711 \\ orrison@hmc.edu \\ David T. Uminsky \\ Boston University \\ Boston, MA 02215 \\ duminsky@math. bu .edu
}

\section{Introduction}

Imagine a survey in which 110 people were given a set $\{A, B, C, D, E\}$ of five items, and were asked to vote for their two favorite items from the set. The data has been presented to you as vector $f \in \mathbb{R}^{10}$ where

$$
f=\left[\begin{array}{c}
2 \\
12 \\
11 \\
6 \\
17 \\
8 \\
4 \\
24 \\
20 \\
6
\end{array}\right] \quad \begin{array}{ll}
A B \\
B C \\
B D \\
C D \\
D E
\end{array}
$$


with the numbers corresponding to the votes for the unordered pairs on the right. For example, two people voted for items $A$ and $B$, while 24 people voted for items $C$ and $D$.

A relatively new starting point for analyzing this sort of data is (generalized) spectral analysis. This is a nonmodel-based approach to the exploratory analysis of data associated with sets, like the set of unorderd pairs above, that have a fair amount of symmetry. It was initially pioneered by Diaconis in $[1,2]$. See also [4].

Should you decide that spectral analysis is worth looking into (as we hope to convince you), then you will be happy to know that there are efficient algorithms for doing spectral analysis $[5,6,7]$. Perhaps more interestingly, at least from a mathematical perspective, these algorithms involve an intriguing mixture of ideas and techniques from linear algebra, abstract algebra, numerical analysis, and graph theory.

The focus of this paper is the linear algebraic framework in which the spectral analysis of voting data like that above is carried out. As we will show, this framework can be used to pinpoint voting coalitions in small voting bodies like the United States Supreme Court. Our goal is to show how simple ideas from linear algebra can come together to say something interesting about voting. And what could be more simple than where our story beginswith counting.

\section{From Counting to Orthogonal Subspaces}

There are 110 people involved in the survey above. The sum of the entries in the vector $f$ is therefore 110 , so the average number of votes given to each of the ten pairs of items is simply $110 / 10=11$. In the long run, this information may or may not be useful, but it seems like a reasonable place to start. After all, if each of the entries in $f$ had been near the average, we could summarize the data by saying that each pair seems just as likely to have been chosen.

After computing the average, our next step might be to compute the number of times an individual item, such as $A$ or $C$, was chosen. This would help us to see if there was an item that was particularly popular or unpopular, regardless of with which item it was paired. Since we would want to do this 
for each item, we could use a matrix for this calculation:

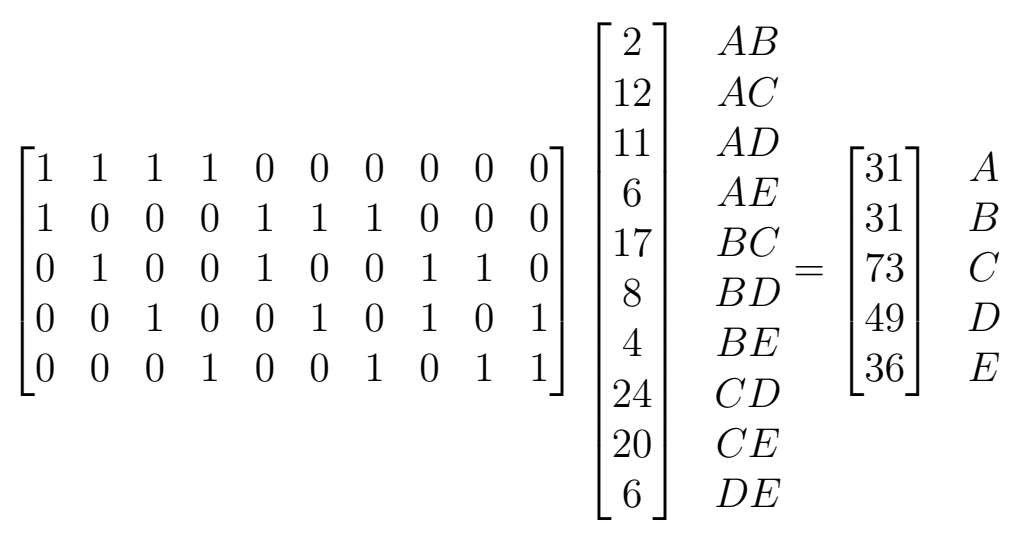

This matrix-vector product shows, for example, that $A$ was chosen $2+12+$ $11+6=31$ times, while $\mathrm{C}$ was chosen $12+17+24+20=73$ times. For reference, we will refer to the $5 \times 10$ matrix used in (1) as $T_{1}$. In other words,

$$
T_{1}=\left[\begin{array}{llllllllll}
1 & 1 & 1 & 1 & 0 & 0 & 0 & 0 & 0 & 0 \\
1 & 0 & 0 & 0 & 1 & 1 & 1 & 0 & 0 & 0 \\
0 & 1 & 0 & 0 & 1 & 0 & 0 & 1 & 1 & 0 \\
0 & 0 & 1 & 0 & 0 & 1 & 0 & 1 & 0 & 1 \\
0 & 0 & 0 & 1 & 0 & 0 & 1 & 0 & 1 & 1
\end{array}\right] .
$$

But why stop with one matrix? For example, the average could have been computed using the matrix

$$
T_{0}=\left[\begin{array}{llllllllll}
\frac{1}{10} & \frac{1}{10} & \frac{1}{10} & \frac{1}{10} & \frac{1}{10} & \frac{1}{10} & \frac{1}{10} & \frac{1}{10} & \frac{1}{10} & \frac{1}{10}
\end{array}\right]
$$

since the product of $T_{0}$ and our data vector is precisely the sum of the entries in the vector divided by 10 . In fact, we could even construct the matrix $T_{2}$ that computes the number of times that each pair was chosen. Of course, this turns out to just be the $10 \times 10$ identity matrix since the data was originally defined in terms of pairs of items. Nonetheless, the matrices $T_{0}, T_{1}$, and $T_{2}$ seem to be just the ticket when it comes to counting. But wait, there's more!

If we define $N_{i}$ to be the nullspace of $T_{i}$, then we get the chain

$$
N_{0} \supset N_{1} \supset N_{2} \text {. }
$$

By definition, however, all of the nonzero counting information is actually contained in the orthogonal complements of the $N_{i}$, which are also the row spaces of the $T_{i}$. This leads to the "complementary" chain

$$
N_{0}^{\perp} \subset N_{1}^{\perp} \subset N_{2}^{\perp} \text {. }
$$


This chain of subspaces makes sense. After all, if we know how many times every pair was chosen, we can figure out the number times each individual item was chosen. This information can then be used to compute the average.

We will refer to the 1-dimensional subspace $N_{0}^{\perp}$ as the mean effects space; it contains all of the information needed to compute the average. The 5dimensional subspace $N_{1}^{\perp}$ is the first order effects space; it contains all of the information needed to compute the number of times a particular item was chosen. Lastly, the 10-dimensional subspace $N_{2}^{\perp}$ is the second order effects space; it is simply the original vector space containing all of the information associated with pairs of items.

Given the chain $N_{0}^{\perp} \subset N_{1}^{\perp} \subset N_{2}^{\perp}$ of subspaces, we could next ask about the effect that each subspace has on our data vector $f$. For example, we could compute the parts of $f$ that are contained in $N_{0}^{\perp}, N_{1}^{\perp}$, and $N_{2}^{\perp}$. Because these subspaces form a chain, however, it is more instructive to compute the parts of $f$ that are introduced as we move up the chain, i.e., to compute what we need to build the vector $f$ as we move from $N_{0}^{\perp}$ to $N_{1}^{\perp}$ to $N_{2}^{\perp}$. This gives rise to an orthogonal decomposition

$$
\mathbb{R}^{10}=M_{0} \oplus M_{1} \oplus M_{2}
$$

of the original vector space, where $N_{0}^{\perp}=M_{0}, N_{1}^{\perp}=M_{0} \oplus M_{1}$, and $N_{2}^{\perp}=$ $M_{0} \oplus M_{1} \oplus M_{2}$. The vector $f$ can therefore be written uniquely as a sum $f=f_{0}+f_{1}+f_{2}$ where $f_{i} \in M_{i}$. For our example, we have

$$
f=\left[\begin{array}{c}
2 \\
12 \\
11 \\
6 \\
17 \\
8 \\
4 \\
24 \\
20 \\
6
\end{array}\right] \quad f_{0}=\left[\begin{array}{l}
11 \\
11 \\
11 \\
11 \\
11 \\
11 \\
11 \\
11 \\
11 \\
11
\end{array}\right] \quad f_{1}=\left[\begin{array}{r}
-26 / 3 \\
16 / 3 \\
-8 / 3 \\
-7 \\
16 / 3 \\
-8 / 3 \\
-7 \\
34 / 3 \\
7 \\
-1
\end{array}\right] \quad f_{2}=\left[\begin{array}{r}
-1 / 3 \\
-13 / 3 \\
8 / 3 \\
2 \\
2 / 3 \\
-1 / 3 \\
0 \\
5 / 3 \\
5 D \\
2 \\
-4
\end{array}\right] \begin{aligned}
& A B \\
& B D \\
& B E \\
& C D \\
& C E \\
& D E
\end{aligned}
$$

The 1-dimensional space $M_{0}$ is still just the mean effects space. The 4dimensional subspace $M_{1}$, however, can be thought of as the space of pure 
first order effects, since we have removed the mean effects contained in $N_{0}^{\perp}$. Likewise, the 5-dimensional subspace $M_{2}$ can be thought of as the space of pure second order effects, since we have removed the mean and first order effects from $N_{2}^{\perp}$.

Now that we have isolated $f_{0}, f_{1}$, and $f_{2}$, the next step might be to explore more deeply the way in which these vectors contribute to the data vector $f$. We could begin by comparing the squared norms of the $f_{i}$. One reason for doing this lies in the fact that, since the $M_{i}$ are orthogonal to each other, we know that

$$
\|f\|^{2}=\left\|f_{0}\right\|^{2}+\left\|f_{1}\right\|^{2}+\left\|f_{2}\right\|^{2} .
$$

By comparing the squared norms, we can therefore get a sense for where the data is concentrated. In our case, $\left\|f_{0}\right\|^{2}=1210,\left\|f_{1}\right\|^{2} \approx 422.67$, and $\left\|f_{2}\right\|^{2} \approx 53.33$. Now the norm of $f_{0}$ captures nothing more than the number of people voting. The relatively large size of $f_{1}$ in comparison to $f_{2}$, however, suggests that the first order effects are contributing heavily to this data.

Before we attempt to pinpoint which item or items from the set $\{A, B, C, D, E\}$ might actually be contributing to $f_{1}$, notice that even though $\operatorname{dim} M_{1}=4$, there are five natural effects to consider, namely the individual effects of each of the five items. In other words, there are too many items to just find a basis vector in $M_{1}$ for each and to then write $f_{1}$ in terms of that basis. As noted in [1], however, there is a straightforward way around this that makes use of inner products.

For each item $x$, consider the function $g_{x}$ which is defined on the unordered pairs of items, and whose value at a pair is 1 if $x$ is in the pair, and 0 otherwise. These functions correspond to the rows of $T_{1}$. We can project each of these functions into $M_{1}$, normalize the projection, and then compute their inner products with a normalized version of the projection $f_{1}$. The resulting numbers, all of which are between -1 and 1 , measure how much the directions of $f_{1}$ and the components of the $g_{x}$ in $M_{1}$ agree. For our data vector $f$, this approach leads to the numbers

\begin{tabular}{|c|c|c|c|c|}
\hline A & B & C & D & E \\
\hline-0.41 & -0.41 & 0.91 & 0.16 & -0.25 \\
\hline
\end{tabular}

which suggest that the respondents in the survey really liked item $\mathrm{C}$ but were slightly averse to choosing items A and B. Indeed, a quick glance back at the original data confirms this. 
We could also compute similar inner products for the pure second order effects. Here the natural functions to consider correspond to the original pairs and the rows of $T_{2}$, with a 1 in just one position and zeros elsewhere. The resulting numbers

\begin{tabular}{|c|c|c|c|c|c|c|c|c|c|}
\hline $\mathrm{AB}$ & $\mathrm{AC}$ & $\mathrm{AD}$ & $\mathrm{AE}$ & $\mathrm{BC}$ & $\mathrm{BD}$ & $\mathrm{BE}$ & $\mathrm{CD}$ & $\mathrm{CE}$ & $\mathrm{DE}$ \\
\hline-0.06 & -0.84 & 0.52 & 0.39 & 0.13 & -0.06 & 0.00 & 0.32 & 0.39 & -0.77 \\
\hline
\end{tabular}

suggest, for example, that the 12 votes for the pair $\{A, C\}$ are due mostly to C's popularity, not the popularity of the pair.

Now for such a small data set, it may seem as though we went to a lot of trouble to end up only saying that "people seem to really like item C." In fact, you may have already come to that conclusion when you first saw the data, or after you saw how many people chose a pair containing $\mathrm{C}$. The point we want to make is that this approach applies to any survey in which people are asked to choose their top $k$ items from a list of size $n$. In fact, if we assume that $0 \leq k \leq n / 2$ (we will ask them to choose their least favorites if we must), then we get an orthogonal decomposition

$$
M=M_{0} \oplus M_{1} \oplus \cdots \oplus M_{k-1} \oplus M_{k}
$$

where $M$ is the underlying $\left(\begin{array}{l}n \\ k\end{array}\right)$-dimensional vector space of real-valued functions defined on the $k$-element subsets of an $n$-element set. The subspace $M_{i}$ captures the pure $i^{\text {th }}$ order effects of the voting data. The projections of a data vector $f \in M$ into each of the $M_{i}$ can be computed with the hope of uncovering hidden large-scale structure. Subsequent inner product calculations can then lead to the uncovering of hidden small-scale structure.

As we will see in the next section, this simple approach to untangling survey data can also be applied to voting data that arises when committees vote "yea" or "nay" on several issues. The trick, perhaps to the delight of committee members everywhere, is to let the issues do all of the voting!

\section{From Surveys to the Supreme Court}

When the members of a committee are asked to vote "yea" or "nay" on an issue, and none of the members abstain from the vote, the result is a splitting of the committee into two groups - the winners (or majority) and the losers 
(or minority). Once we know either group, we automatically know the other, so for convenience, we will focus on the minority members for each issue.

Now although the committee members are really doing all of the voting, we can turn the tables by pretending as though the issues are actually voting on the subset of members that it wants to make up the minority when it comes before the committee. In this way, we can use the issues and their "votes" to try to pinpoint coalitions in the committee. Moreover, to analyze the resulting data, we separate it into different functions, one for each of the possible number of members in the minority. We then analyze each of the functions using the techniques described in the earlier sections. (See [3] for more details.)

As a proof of concept, consider the well-studied nine member "committee" of justices on the United States Supreme Court, say from 1994 to 1998. For each case (issue) in which there are no abstentions, there can be zero, one, two, three, or four justices that form the minority. We limit our analysis to non-unanimous cases, and for the Supreme Court buffs out there, we have also limited our analysis to the cases in which a signed opinion was issued. Our data comes from the database maintained by Spaeth [8], and the results of our analysis are summarized in Table 1.

As we will see, after looking at the results in the table, it is easy to go back and find the information that supports it. We want to stress, however, that finding the most important coalitions in a committee would be labor intensive and unsystematic without something like spectral analysis. This would be especially true if you were starting with raw data and knew essentially nothing about the committee members. Moreover, as described in [5, 6], by using a combination of ideas and techniques from introductory courses in linear algebra, abstract algebra, numerical analysis, and graph theory, the results presented in Table 1 can be computed very efficiently.

\section{Cases with 8-1 splits}

There were 37 cases which split 8-1. The lone dissenter was Stevens 29 times, Thomas wrote 3 such dissents, neither Ginsburg nor Breyer ever dissented on their own, and the remaining five justices each wrote one lone dissent. Not surprisingly, the first line of the table show that the 8-1 data points strongly in the direction of Stevens dissenting. 
Table 1: Rehnquist Court 1994-1998, 192 non-unanimous cases

\begin{tabular}{|c|c|c|c|c|c|c|c|c|c|c|}
\hline split & subspace & norm $^{2}$ & \multicolumn{8}{|c|}{ four largest (using absolute value) inner products } \\
\hline $8-1$ & $M_{1}$ & 703 & St & .996 & $\mathrm{Br}$ & -.165 & Gi & -.165 & Sc & -.125 \\
\hline $7-2$ & $M_{2}$ & 183 & ThSc & .732 & StGi & .476 & StBr & .354 & StSc & -.330 \\
\hline $7-2$ & $M_{1}$ & 59 & St & .695 & 0 & -.452 & So & -.348 & $\mathrm{Ke}$ & -.348 \\
\hline $6-3$ & $M_{3}$ & 72 & ReThSc & .647 & $0^{\prime}$ ThSc & .345 & BrThSc & -.321 & $\operatorname{Re} 0^{\prime} \mathrm{Sc}$ & -.290 \\
\hline $6-3$ & $M_{2}$ & 105 & ThSc & .626 & StGi & .345 & GiTh & -.309 & ReTh & .237 \\
\hline $6-3$ & $M_{1}$ & 16 & Th & .656 & $\mathrm{Sc}$ & .540 & $\mathrm{Ke}$ & -.501 & $\mathrm{Br}$ & -.270 \\
\hline $5-4$ & $M_{4}$ & 316 & StGiBrSo & .954 & KeReThSc & .344 & O'ReThSc & .341 & StBrSoSc & -.269 \\
\hline $5-4$ & $M_{3}$ & 199 & StBrSo & .379 & StGiSo & .368 & GiBrSo & .293 & StGiBr & .293 \\
\hline $5-4$ & $M_{2}$ & 360 & StSo & .315 & $\mathrm{StBr}$ & .301 & ThSc & .282 & StGi & .265 \\
\hline $5-4$ & $M_{1}$ & 22 & $\mathrm{Ke}$ & -.646 & So & .418 & $0^{\prime}$ & -.380 & Gi & .380 \\
\hline
\end{tabular}

St Stevens Gi Ginsburg Br Breyer So Souter Ke Kennedy

0' O'Connor Re Rehnquist Th Thomas Sc Scalia

\section{Cases with 7-2 splits}

There are first and second order effects for the cases which split 7-2. In this case, the squared norm of the projection onto $M_{2}$ is 183 , while the squared norm of the projection onto $M_{1}$ is 59 . This portion of the data is therefore dominated by the pure second order effect.

The pure second order effect points in the direction of Thomas-Scalia dissenting, but also has noticeable components in the Stevens-Ginsburg and Stevens-Breyer directions. The pure first order effect, although not as strong as the pure second order effect, points in the direction of Stevens dissenting.

In this portion of the data, there are 48 cases with 7-2 splits, where 11 of them are Thomas-Scalia, 9 are Stevens-Ginsburg, and 8 are Stevens-Breyer dissenting. Of the 48 cases with pairs dissenting, Stevens is a dissenter in 24 of them. In addition to Ginsburg and Breyer, Stevens dissents with Souter, Kennedy, and Thomas. 


\section{Cases with 6-3 splits}

For the 6-3 cases, the second order effect is the largest with a squared norm of 105. The largest inner product for the pure second order effects corresponds to Thomas-Scalia dissenting. Moreover, the other projections for the 6-3 cases also point in this general direction.

The largest pure first order effect is Thomas dissenting, and the next largest is Scalia dissenting. The two largest third order effects are ThomasScalia dissenting joined first by Rehnquist, and then by O'Connor. The negative sign on the Breyer-Thomas-Scalia triple suggests that these three justices seldom dissent together in a 6-3 split.

Again, when we examine the data we find that this is a good summary. Of the 44 cases with a 6-3 split, Thomas and Scalia join together in dissent in 22 of them. They are joined ten times by Rehnquist, seven times by O'Connor, three times by Kennedy, and once each by Souter and Stevens.

\section{Cases with 5-4 splits}

The pure second order effect is the largest for the 5-4 cases with a squared norm of 360. The five largest inner products for the pure second order effect are relatively close to each other. They correspond to "liberal" dissents (Stevens-Souter, Stevens-Breyer, and Stevens-Ginsburg) and "conservative" dissents (Thomas-Scalia and Rehnquist-Scalia, the latter of which is not in the table).

The pure fourth order effect is the next largest with a squared norm of 316 . The pure fourth order effect is in the "liberal" dissenting direction (StevensGinsberg-Breyer-Souter) with smaller components in the "conservative" dissenting directions (Rehnquist-Thomas-Scalia-Kennedy and Rehnquist-ThomasScalia-O'Connor). This seems to fit the data well. For the 63 cases with 5-4 splits, 28 cases are dissents by Stevens-Ginsberg-Breyer-Souter, eight are Rehnquist-Thomas-Scalia-O'Connor, and seven are Rehnquist-ThomasScalia-Kennedy.

Now although the pure first order effects are the smallest, they have an interesting interpretation. The "swing" voter Kennedy has a large significant negative value (-.646) suggesting that he rarely ends up in the minority in 5-4 splits, which is what would be expected of swing voters. The case for O'Connor, while smaller, is similar. 


\section{Conclusion}

Spectral analysis is a powerful tool for doing exploratory data analysis, and given that efficient algorithms for doing this type of analysis exist [5, 6, 7], political scientists, economists, and market research analysts seem to have every incentive to include it in their arsenal. As we noted in the introduction, spectral analysis was initially pioneered by Diaconis in $[1,2]$. The interested reader is strongly encouraged to delve into these sources, both of which are teeming with tantalizing open questions and deep ideas.

\section{Acknowledgments}

We gratefully acknowledge a Harvey Mudd College Beckman Research Award which supports research by undergraduates with faculty. We would like to thank the Charles Phelps Taft Fund at the University of Cincinnati. Special thanks also to Jon Jacobsen and Francis Su for helpful comments and suggestions.

\section{References}

[1] P. Diaconis, Group representations in probability and statistics, Institute of Mathematical Statistics, Hayward, CA, 1988.

[2] P. Diaconis, A generalization of spectral analysis with application to ranked data, Ann. Statist. 17 (1989), no. 3, 949-979.

[3] M. Iwasaki, Spectral analysis of multivariate binary data, J. Japan Statist. Soc. 22 (1992), no. 1, 45-65.

[4] J. Marden, Analyzing and modeling rank data, Monographs on Statistics and Applied Probability, vol. 64, Chapman \& Hall, London, 1995.

[5] D. Maslen, M. Orrison, and D. Rockmore, Computing isotypic projections with the Lanczos iteration, SIAM Journal on Matrix Analysis and Applications 25 (2004), no. 3, 784-803.

[6] M. Orrison, An eigenspace approach to decomposing representations of finite groups, Ph.D. thesis, Dartmouth College, 2001. 
[7] D. Rockmore, Some applications of generalized FFTs, Groups and computation, II (New Brunswick, NJ, 1995), Amer. Math. Soc., Providence, RI, 1997, pp. 329-369.

[8] H. Spaeth, UNITED STATES SUPREME COURT JUDICIAL DATABASE, 1953-1997 TERMS [Computer file]. 9th ICPSR version. East Lansing, MI: Michigan State University, Dept. of Political Science [producer], 1998. Ann Arbor, MI: Inter-university Consortium for Political and Social Research [distributor], 1999. 